\title{
In Vitro to Human In Vivo Translation - Pharmacokinetics and Pharmacodynamics of Quinidine
}

\author{
Sebastian Polak ${ }^{1,2}$ \\ ${ }^{1}$ Unit of Pharmacoepidemiology and Pharmacoeconomics, Faculty of Pharmacy, Jagiellonian University Medical College, \\ Krakow, Poland; ${ }^{2}$ Simcyp Limited, a Certara Company, Blades Enterprise Centre, Sheffield, UK
}

\begin{abstract}
Summary
The translational sciences aim to transfer results from basic research to the treatment of animals or patients. One of the approaches that could be utilized to achieve this goal is the in vitro-in vivo extrapolation (IVIVE) of pharmacokinetic (PK) and pharmacodynamic $(P D)$ properties using in silico methods. Such methodology, if properly applied, could help substantially reduce the use of animals in pre-clinical research. Here, quinidine was chosen as an example of a drug with cardiac effects and results of nine published clinical studies describing its $P K$ (plasma concentration) and $P D(Q T c B / \triangle Q T c B)$ effects were mimicked by combination of the IVIVE platform Simcyp (pharmacokinetics prediction) with the ToxComp (cardiac effect prediction) system, based exclusively on in vitro data. The results show that reliable QT prediction is possible using the mechanistic IVIVE of the PK and PD effects. This can be considered a proof-of-concept that also could be applied as a drug safety evaluation procedure.
\end{abstract}

Keywords: modeling and simulation, drug cardiotoxicity, quinidine, IVIVE

\section{Introduction}

The scope of translational science ranges from a general description of the drug and medical device development process from bench to bedside, through translating research into practice, culminating in the complex approach where the multidisciplinary collaboration of translational science accelerates the specific scientific application (Woolf, 2008; Zerhouni, 2005). Translational science divides the drug development process into a series of incremental steps ${ }^{1}$. Regardless of the translational step under consideration, all the stages contribute to a more effective use of the available information, and thus an efficient transfer of the developed therapies from the bench to the bedside.

In vitro-in vivo extrapolation (IVIVE) of pharmacokinetic (PK) and pharmacodynamic (PD) properties of a drug by in silico methods provides a model-based drug development method that facilitates progression to the bedside endpoint (Lalonde et al., 2007). The PK and PD models used in this study are mechanistic models working exclusively on the in vitro data, and therefore no clinical study data was used at the results simulation level to fit parameters and improve the prediction. The mechanistic models are widely utilized in the "PK arena" and are becoming more prominent in the "PD arena" and vital areas of toxicology and drug safety (Marshall et al., 2006). This study unites the concepts of PK and PD mechanistic modeling and simulation to highlight the importance of assessing drug effect and safety in the preliminary phases of the drug development process. The IVIVE application approach necessitates the provision of three data sets: 1) drug related (ADME processes and activity), 2) system data (describing population and variability of the chosen parameters), and 3) simulated trial design (Rostami-Hodjegan and Tucker, 2007). The IVIVE methodology is a robust evaluation tool that assesses inter-individual variability based on the virtual population characteristics in the population study group.

To assess the application value of the described approach, quinidine was selected as the model drug in the virtual study described here. The study endpoints covered plasma concentration of the parent compound and its main metabolite, $3-\mathrm{OH}$ quinidine, from the pharmacokinetic side. Either QTc interval or the drug triggered change as compared to the baseline $(\Delta \mathrm{QT} /$ $\Delta \mathrm{QTc}$ ) were used as the pharmacodynamic effect descriptors. This pharmacodynamic effect dictates that drug cardiac safety should be regarded as a pivotal focal point of this study. Preclinical studies routinely use in vitro approaches to assess cardiac safety; however, non-rodent species (e.g., dogs, monkeys) are commonly used in the assessment procedure. This study proposes a novel concept based on a combination of mechanistic PBPK/PD modeling and simulation to predict the cardiac effects of drugs and thus help to incorporate the 3Rs concept

Received November 30, 2012; accepted in revised form February 4, 2013

1 http://www.tuftsctsi.org/About-Us/What-is-Translational-Science.aspx 
into practice, either by waiving or reducing animal studies.

The aim of this study was to use available in vitro data exclusively to simulate the in vivo effects of drugs. Both arms of a clinical study, i.e., pharmacokinetics and pharmacodynamics, were mimicked. The focus of the study was to assess the interindividual variability and to establish an accurate simulation methodology.

\section{Materials and methods}

\section{Data}

A wide literature search to find papers describing combined pharmacokinetic and pharmacodynamic effects of quinidine was performed. The study inclusion criteria were: a) healthy Caucasian volunteers, b) availability of information about the quinidine pharmacokinetics, ideally presented as a drug plasma concentration change in time, c) PD results presented as QT/ QTc or $\Delta \mathrm{QT} / \Delta \mathrm{QTc}$ (regardless of the correction type), thus comparable with the simulation outputs. Nine papers fulfilling such conditions were identified and used for the study (Belz et al., 1982; Ching et al., 1991; El-Eraky and Thomas, 2003; Fieldman et al., 1977; Kaukonen et al., 1997; Laganiere et al., 1996; Min et al., 1996; Olatunde and Price Evans, 1982; Shin et al., 2007). Characteristics of the clinical studies derived from the identified papers are presented in Table 1.

When applicable, data from the selected manuscripts were used directly or were derived from the graphs after digitization. The latter was done using the GetData Graph Digitizer tool (http://www.getdata-graph-digitizer.com).

\section{Simulation study}

All simulations were performed using two complementary software programs - Simcyp platform version 12 for the in vitro-in vivo extrapolation of the ADME processes (http://www.simcyp. com) and ToxComp version 1.6 (http://www.tox-portal.net) for cardiac effect prediction.
Simcyp

The population-based Simcyp Simulator streamlines drug development through the modeling and simulation of pharmacokinetics (PK) and pharmacodynamics (PD) in virtual populations. The Simcyp Simulator is the platform for the prediction of pharmacokinetic outcomes in clinical populations with the use of the human physiology, genetics, and epidemiology information. Integration of this information with in vitro data allows the prediction of PK drug behavior in "real-world" populations. The Simcyp Simulator also can identify key pre-clinical data requirements, which are extremely valuable for redefining and optimizing early drug development processes and procedures.

\section{ToxComp}

ToxComp is a user-friendly, systems biology driven, modeling and simulation based platform for the proarrhythmic potency assessment of chemicals at the population level. The system utilizes the in vitro-in vivo extrapolation approach, thus by default the input data comes from in vitro ionic current inhibition studies (Polak et al., 2012a). The model describing the electrophysiology of the human left ventricular cardiomyocyte, applied in the current study, was based on the work reported by ten Tusscher et al. (2004), with minor modifications. The reasoning behind this selection was that the majority of the data used for the model development was of human origin (Niederer et al., 2009). The ToxComp system contains a module for the virtual population development that is used subsequently as a basis for the simulation. Randomly picked individuals carry unique demographic and physiological characteristics; the specific parameters include, inter alia: cardiomyocyte area, electric capacitance, and volume (all parameters are age dependent), plasma ion concentration, left ventricle heart wall thickness and heart rate, with the latter following the circadian rhythm (Polak and Fijorek, 2012; Polak et al. 2012b). An additional parameter is the genetic status described by potential modification of the hERG potassium channel gating parameters (Glinka and Polak, 2012). Stable version 1.2 currently is available at http://www.

Tab. 1: Characteristics of the clinical study data used for the simulation

\begin{tabular}{|c|c|c|c|c|}
\hline Reference & $n(M / F)$ & Age (years) ${ }^{*}$ & Study endpoints & Dosing** \\
\hline El-Eraky and Thomas, 2003 & $48(27 / 21)$ & $33 \mathrm{M} / 31 \mathrm{~F}$ & $\triangle \mathrm{QTcB}$ & Oral (3 mg/kg) \\
\hline Fieldman et al., 1977 & $7(5 / 2)$ & $23-48$ & QTcB, $\triangle \mathrm{QTcB}$ & Oral (400 mg) \\
\hline Olatunde and Price Evans, 1982 & $7(4 / 3)$ & $24.9(4.76)$ & QTcB, $\triangle Q T c B$ & Oral (400 mg) \\
\hline Shin et al., 2007 & $13(7 / 6)$ & $26.2(7.5) \mathrm{M} / 27.7$ (3.6)F & QTcB & IV infusion (4 mg/kg) \\
\hline Kaukonen et al., 1997 & $9(4 / 5)$ & $25(4)$ & QTcB & Oral (100 mg) \\
\hline Min et al., 1996 & $12(12 / 0)$ & $23(4.3)$ & $\triangle \mathrm{QTcB}$ & Oral (400 mg) \\
\hline Belz et al., 1982 & $6(6 / 0)$ & $23-33$ & QTcB & Oral (500 mg) \\
\hline Laganiere et al., 1996 & $12(12 / 0)$ & $24(5)$ & QTcB & Oral (200 mg) \\
\hline Ching et al., 1991 & $8(8 / 0)$ & $22-29$ & $\triangle \mathrm{QTcB}$ & Oral (400 mg) \\
\hline
\end{tabular}

*average (SD) where available or range; ** single dose in all cases 
tox-comp.net, either for download or for a live run. This version of the platform is freely available and distributed under the GNU GPLv3 license.

Pharmacokinetic simulation preceded the heart electrophysiological simulation, and during the latter simulation the same group of individuals, carrying the demographic and physiological parameters, were involved in the drug pharmacodynamics simulation together with the predicted drug plasma concentration for all virtual individuals involved in the study.

\section{Input data and modeling assumptions}

The utilized drug-related input data included two elements, i.e., the in vitro information, describing the ADME processes used to run the Simcyp simulation, and the in vitro data describing inhibition of various cardiac ionic currents. For the PK simulation, default quinidine (parent) and 3-OH quinidine (main metabolite) compound files were utilized. The major ADME parameters are listed in Table 2, and their values are presented in the supplementary materials at www.altex-edition.org.

The in vitro inhibition of various cardiac ionic currents was taken from the literature. If this data was not available, it was predicted with previously developed and described QSAR models (Polak et al., 2011, 2012c,d; Wisniowska et al., 2012). It was assumed and confirmed in a subsequent QSAR based simulation that 3-OH quinidine also inhibits ionic currents. As multiple results from various sources were available, those best mimicking the human physiology were selected. The $\mathrm{IC}_{50}$ and $\mathrm{n}$ values are the parameters of the Hill equation used to describe the drug triggered ionic current modifications. The specific equation, part of the ten Tusscher model, describing the current of interest was multiplied by the inhibition factor calculated with the use of the Hill equation (Equation 1).

Equation 1:

$$
\begin{aligned}
& \text { Inhibition Factor } \\
& =\frac{1}{1+\left(I_{50} / \text { DRUG CONCENTRATION }\right)^{n}}
\end{aligned}
$$

where:

IC 50 - concentration at which the ionic current is inhibited by $50 \%$

$n$ - Hill equation parameter

DRUG CONCENTRATION - active drug concentration [ $\mu \mathrm{M}$ ]

Total inhibition was the sum of inhibitions of ionic currents triggered by both drugs - quinidine and its metabolite. Table 3 presents the relevant information for both drugs.

The above listed input parameters, which were used to feed the appropriate QSAR model, were selected to match the parameters used during the measurement of different currents. It was assumed that neither quinidine nor 3-OH quinidine influence human physiological parameters, including plasma ion concentration and heart rate. Two different scenarios were tested where either the total, or unbound, concentrations of both compounds were used for the cardiac electric effect simulation and compared with the clinically observed data for the pharmacody-

\begin{tabular}{|c|c|c|c|}
\hline Compound & Module & Model utilized & Model parameters \\
\hline \multirow{6}{*}{ Quinidine } & Binding & $\begin{array}{l}\text { B/P (blood-to-plasma ratio), } \\
\text { fu (fraction unbound in plasma) }\end{array}$ & Default Simcyp values used \\
\hline & Absorption & ADAM model for oral solid formulations & Solid formulation where applicable \\
\hline & Distribution & Minimal PBPK model & $\begin{array}{l}\text { Poulin and Theil method with the Bierezhkovski } \\
\text { correction }\end{array}$ \\
\hline & Metabolism & Enzyme kinetics & HLM based data for 3-hydroxylation and $\mathrm{N}$-oxidation \\
\hline & Elimination & Renal clearance & $\begin{array}{l}\text { Typical renal clearance for a } 20 \text { - to } 30 \text { - year-old } \\
\text { healthy male }(\mathrm{L} / \mathrm{h})\end{array}$ \\
\hline & Transport & $\begin{array}{l}\text { Transporter kinetics on intestinal } \\
\text { absorption }\end{array}$ & Jmax/Km for the apical efflux ABCB1 transporter \\
\hline \multirow{4}{*}{ 3-OH quinidine } & Binding & $\begin{array}{l}\text { B/P (blood-to-plasma ratio), } \\
\text { fu (fraction unbound in plasma) }\end{array}$ & Default Simcyp values used \\
\hline & Distribution & Minimal PBPK model & $\begin{array}{l}\text { Poulin and Theil method with the Bierezhkovski } \\
\text { correction }\end{array}$ \\
\hline & Metabolism & In vivo clearance & Default Simcyp CLpo value used \\
\hline & Elimination & Renal clearance & $\begin{array}{l}\text { Typical renal clearance for a } 20 \text { - to-30 year-old } \\
\text { healthy male }(\mathrm{L} / \mathrm{h})\end{array}$ \\
\hline
\end{tabular}

Tab. 2: Drug related in vitro ADME data used for quinidine and its main metabolite (3-OH quinidine) in the simulation 
Tab. 3: Drug related in vitro data describing cardiac current inhibition for quinidine and its main metabolite (3-OH quinidine)

\begin{tabular}{|c|c|c|c|c|}
\hline Ionic current & Quinidine $\left[\mathrm{IC}_{50}\right] / \mathrm{n}$ & $\begin{array}{l}\text { Reference and/or } \\
\text { model input parameters }\end{array}$ & $\begin{array}{l}\text { 3-OH quinidine } \\
{\left[\mathrm{IC}_{50}\right] / \mathrm{n}}\end{array}$ & $\begin{array}{l}\text { Reference and/or model } \\
\text { input parameters }\end{array}$ \\
\hline $\mathrm{I} K r$ & $0.82 / 1$ & Kirsch et al., 2004 & $1.19 / 1^{*}$ & $\begin{array}{l}\text { Polak et al., 2012a: } \\
\text { Cell: HEK } \\
\text { Temp: phys } \\
\text { t1: } 2 \mathrm{~s} \\
\text { t2: } 2 \mathrm{~s} \\
\mathrm{~K}^{+} \text {conc: } 5.4 \mathrm{mM} \\
\text { Holding: }-80 \mathrm{mV} \\
\text { Depolarization: } 0 \mathrm{mV} \\
\text { Measurement: } 50 \mathrm{mV}\end{array}$ \\
\hline $\mathrm{I}_{\mathrm{Ks}}$ & $44 / 1$ & Kang et al., 2001 & $39.67 / 1^{*}$ & $\begin{array}{l}\text { Polak et al., 2012c: } \\
\text { Cell: HEK (LQT/minK) } \\
\text { Temp: phys } \\
\text { t1: } 2 \mathrm{~s} \\
\text { Measurement: } 20 \mathrm{mV}\end{array}$ \\
\hline $\mathrm{I}_{\mathrm{CaL}}$ & $10 / 1$ & Michel et al., 2002 & $26.38 / 1^{*}$ & $\begin{array}{l}\text { Wisniowska et al., 2012: } \\
\text { Cell: Rat VM } \\
\text { Temp: phys } \\
\text { t1: } 0.1 \mathrm{~s} \\
\mathrm{Ca}^{2+} \text { conc: } 1.8 \mathrm{mM} \\
\text { Holding: }-40 \mathrm{mV} \\
\text { Depolarization: } 0 \mathrm{mV}\end{array}$ \\
\hline INa,peak & $16.6 / 1$ & Mirams et al., 2011 & - & - \\
\hline
\end{tabular}

${ }^{*}$ QSAR predicted ( $\mathrm{n}$ - assumed to be 1$)$

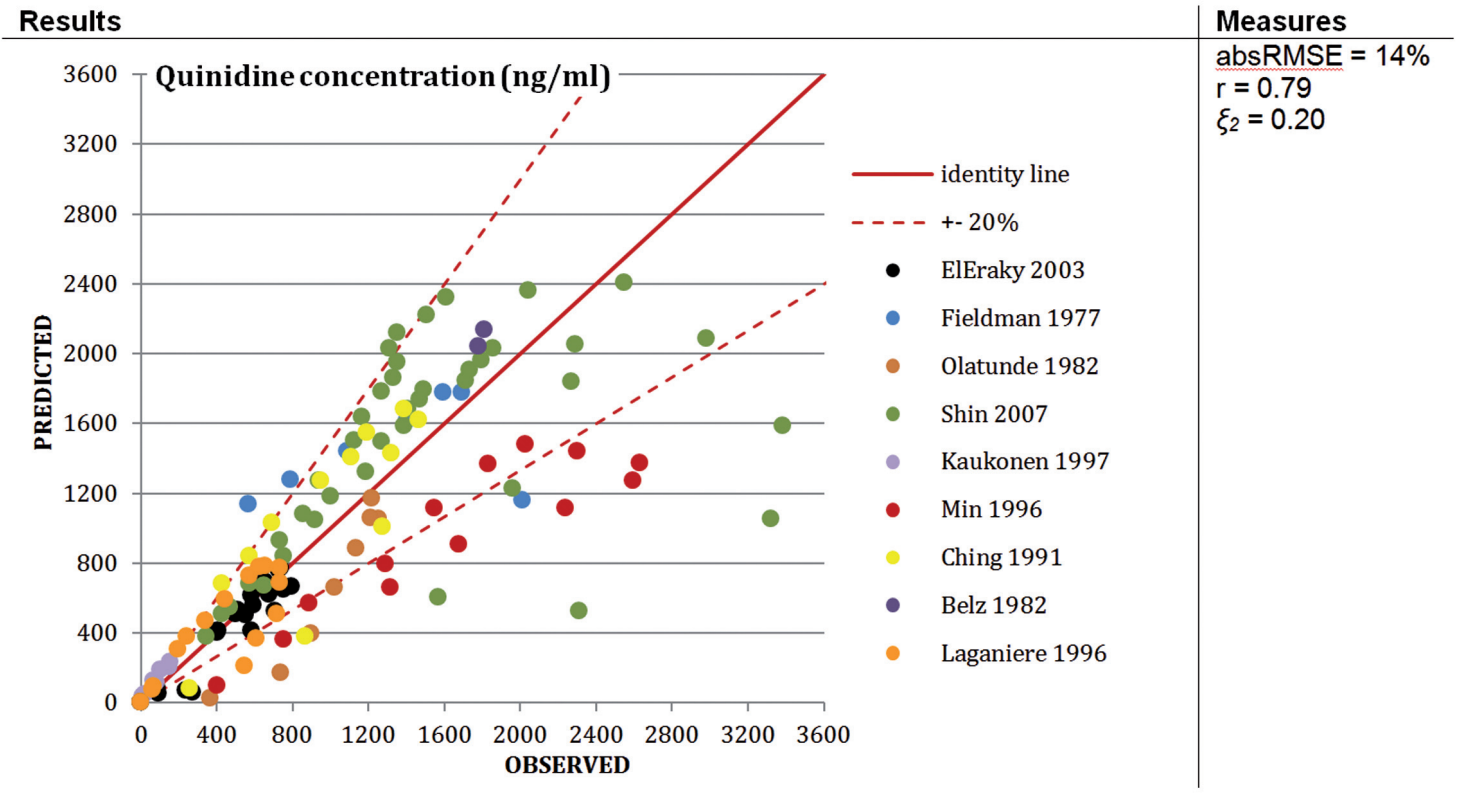

Fig. 1: Plasma concentration prediction

Observed vs. predicted graph and goodness of prediction measures. 
Fig. 2: Pharmacodynamic endpoints $\triangle Q T C$ and QTc for free and total plasma drug concentration respectively Observed vs. predicted graphs and goodness of prediction measures.

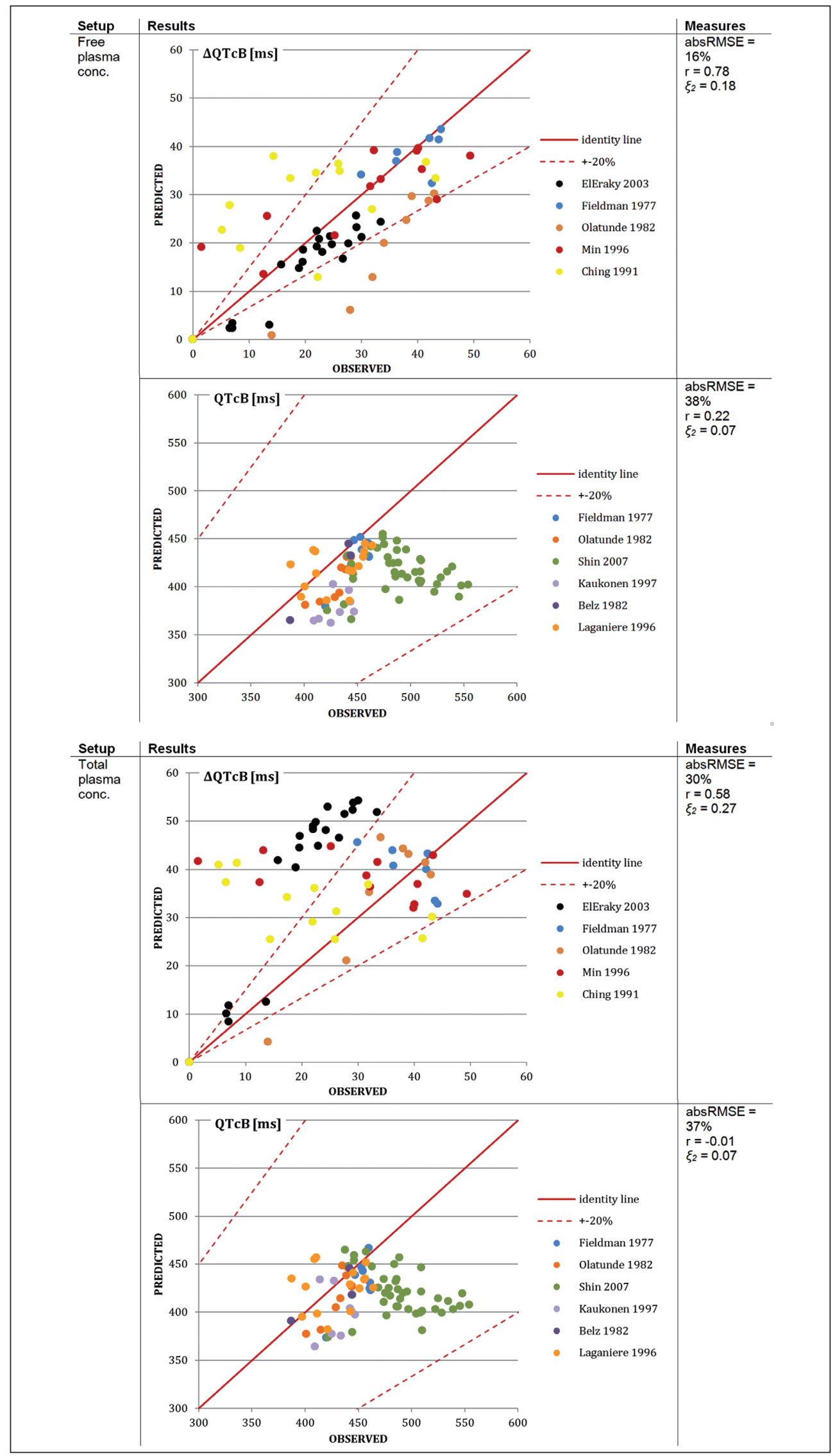


Fig. 3: Total (PRED total) and unbound (PRED free) drug concentration based prediction vs. the observed (OBS) $\Delta Q T c$ values

Effect over time expressed as the average values $( \pm$ SEM). (continued on next page).

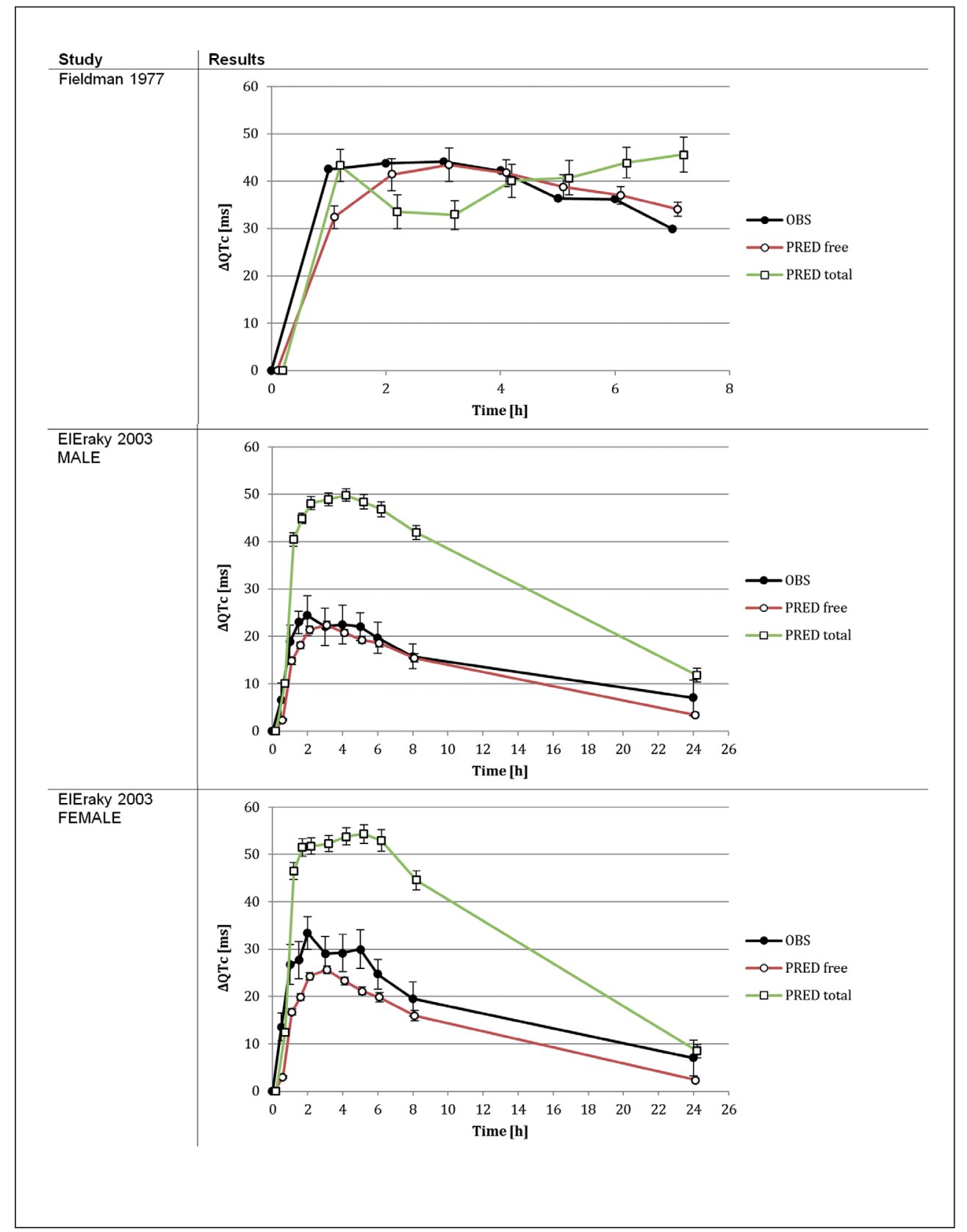

namic endpoints. For all virtual studies the sampling time points were repeated, as in the simulated clinical study. If the time of day the study commenced was provided, the simulation was set to start at the same time. In all other situations, the virtual study was assumed to start at 8:00 a.m.

\section{Output and data analysis}

Results are presented in the form of the observed versus predicted graphs for the pharmacokinetic (drug plasma concentration) and pharmacodynamic (either QTc or $\Delta$ QTc with Bazzet correction applied, or both where applicable) effects. The goodness of prediction measures included absolute RMSE (absRMSE - root mean squared error over difference between maximal and minimal observed value currently analyzed),
Pearson correlation coefficient $r$ and Rescigno $\xi_{2}$ index in accordance with the formula presented in Equation 2 (Rescigno, 1992).

Equation 2:

$$
\xi_{2}=\left(\frac{\sum_{j=1}^{n} \omega_{j}\left[c_{r}\left(t_{j}\right)-c_{x}\left(t_{j}\right)\right]^{2}}{\sum_{j=1}^{n} \omega_{j}\left[c_{r}\left(t_{j}\right)+c_{x}\left(t_{j}\right)\right]^{2}}\right)^{1 / 2}
$$

where:

$\omega j$ - weight (for this study assumed to be 1)

cr $\left(t_{j}\right)$ - observed value (i.e., plasma concentration) in time $t$

$c x\left(t_{j}\right)$ - predicted value (i.e., plasma concentration) in time $t$ 
Fig. 3: (continued from previous page).

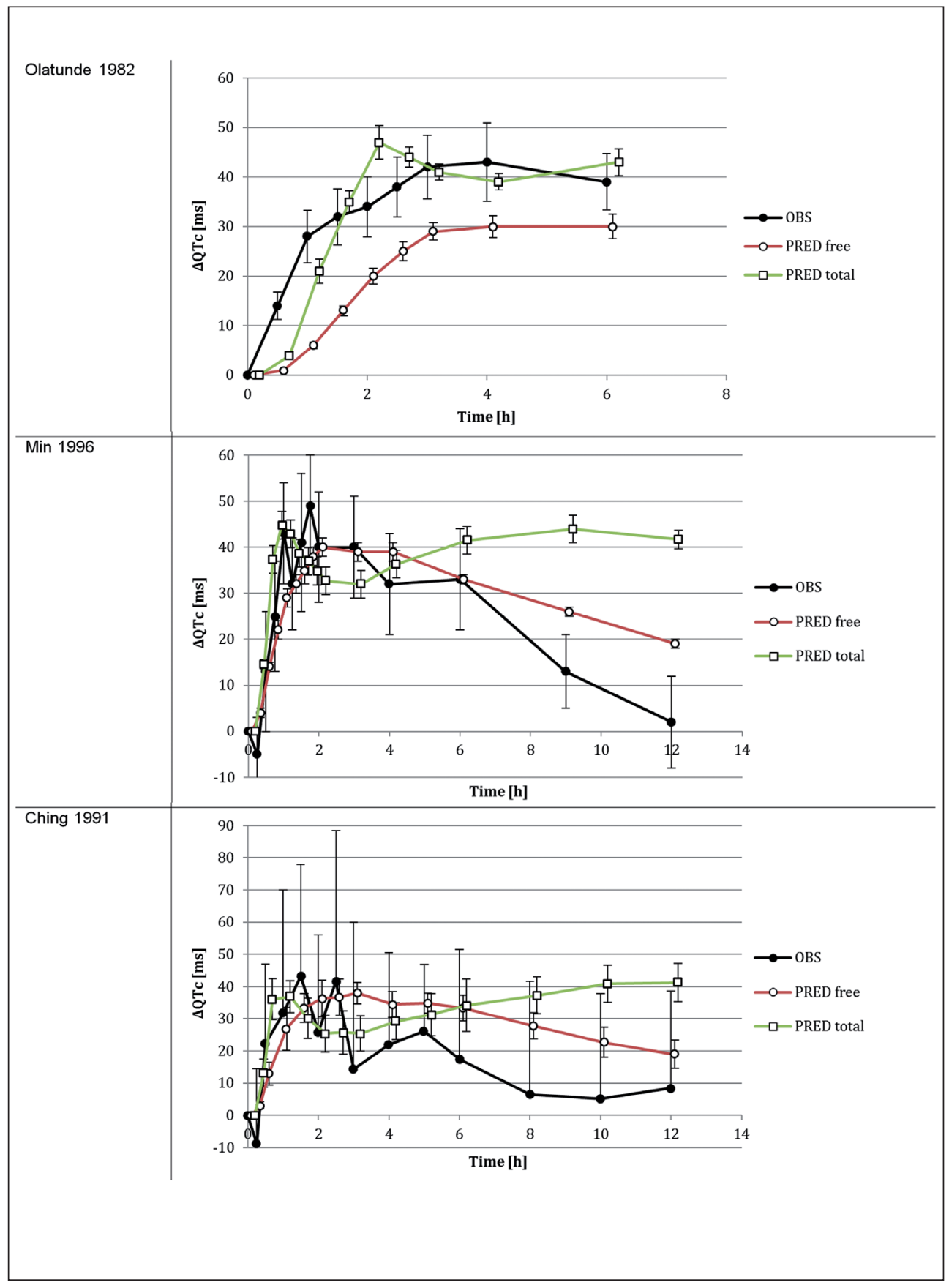

The latter measure is commonly used in the result analysis of bioequivalence studies but was applied in this study as a useful measure enabling the comparison of the two curves (in this case - predicted and observed for quinidine concentration and $\mathrm{QTc} / \Delta \mathrm{QTc}$ change over time).

\section{Results}

Results presented in Figure 1 contain data from all studies presented together as a comparison. The observed vs. predicted graph for quinidine plasma concentration (all studies) is linked with the appropriate goodness of prediction measures. Please refer to the supplementary materials at www.altex-edition.org for the individually presented, detailed results containing an additional set of information.

Figure 2 presents the $\triangle \mathrm{QTcB}$ and QTcB simulation results (5 and 6 studies respectively) compared with observed values presented in a similar format as above.

The clinical endpoints were compared directly with the predicted values for all time points characteristic of the clinical study protocols. Figure 3 presents a comparison between the observed and predicted $\Delta \mathrm{QTc}$ calculated values based on the free and total compound concentrations. 


\section{Discussion}

The study was conducted to test the ability to simulate the in vivo activity of drugs based exclusively on in vitro data, mimicking both the pharmacokinetic and pharmacodynamic arms of a clinical study. According to this assumption, the simulation results are presented as both the plasma concentration and QTc/ $\Delta$ QTc change over time. It is worth noting that the endpoint choice for a particular simulated study depends exclusively on the data presented in the original paper. Comparison between the predicted and observed values of the heart rate corrected QT intervals gives more information relating to the quality of the human left ventricular myocyte electrophysiology model and its ability to mimic human electrophysiology. $\triangle \mathrm{QTc}$ is more model-independent and gives information about the ability of the model to react to factors influencing the QT lengths (in our case - drugs).

Considering that both systems, namely Simcyp for the PK simulation and ToxComp for the PD simulation, utilized no in vivo clinical data, with the exception of the $3-\mathrm{OH}$ quinidine CLpo value, which could be replaced either by the whole organ metabolic clearance scaled from the in vitro systems or in vitro measured enzyme kinetics, the obtained results are consistent with the clinically observed data.

Analysis of the clinical study results shows one characteristic feature, namely the high heterogeneity of the results, even for the studies with relatively similar protocols. The observed differences could be a result of the various analytical methods applied, sampling times, etc., and may not necessarily reflect the real variability. This is an important factor influencing the predictivity of the applied methods, which have a tendency to underpredict the clinical results. Such an effect is not obvious for the PK endpoint prediction (plasma concentration) and $\triangle \mathrm{QTC}$ from the PD perspective, but it seems to be systematic for the QTc measure. The explanation for this difference lies in the characteristics of the model used to describe human cardiac myocyte electrophysiology, which tends to underpredict the cardiomyocyte action potential duration. This was accounted for at the ToxComp system planning level where the focus was put on mimicking the human situation and the ten Tusscher approach (ten Tusscher et al., 2004) offered a suitable model. However, there are novel models based exclusively on human data, which can help with the under ion issue, and their application is planned for the future (O'Hara and Rudy, 2011).

As previously mentioned, the verification of viable simulation methodology was one of the additional study goals. The author is confident that this is the first published study where the utilized methods and techniques were applied in parallel. From a practical perspective, the main concern is applying an accurate operational drug concentration. Two major elements need to be considered - drug target (plasma concentration is a surrogate for the drug meeting the ionic channels in the heart) and binding (free or total concentration). The extracellular water in the heart tissue is regarded as the drug target and hence the preferred location for measuring the active drug concen- tration. However, considering that the vascular wall is not an efficient barrier, the measurement of plasma drug concentration is a realistic and acceptable surrogate. There are some drugs (physico-chemical character) and/or pathophysiological (i.e., arteriosclerosis triggered changes in the vascular wall permeability) factors that could potentially influence the balance and change the plasma-to-heart tissue extracellular water concentration ratio. Moreover, of greater importance from the in silico realized in vitro-in vivo extrapolation perspective is to consider the unbound drug as active (driving the pharmacological effect).

In this study both options, total and unbound plasma concentrations, were tested and the results are presented. The quality of prediction, based on a visual check and the goodness of prediction measures analysis, is more accurate for the free plasma concentration study arm. Graphical inspection demonstrates that for the free concentration setup, either most $(\triangle \mathrm{QTcB})$ or all of the QTcB values for the various time points deviate only $\pm 20 \%$ from the identity line range. Simulations run in parallel using the total plasma concentration as the operational concentration significantly overpredicted the cardiac effect. As this cannot be considered as proof, additional analysis was performed where the observed PD endpoints were directly compared with the predicted values for all time points characteristic of the clinical studies protocol. The graphs presented in Figure 3 verify the hypothesis and show the problems associated with the suitable mimicking of the effect over time curve shape.

For the El-Eraky study (El-Eraky and Thomas, 2003), it was noted that the switch from free to total concentration results in a drastic increase of the predicted $\triangle \mathrm{QTC}$ values. Considering the relatively low drug concentration values, an increase of these values results in a significantly larger $\mathrm{I}_{\mathrm{Kr}}$ current inhibition as compared to higher concentrations observed in other studies, as the concentration points lie on the slope of the Hill equation where maximum in vitro change is observed. This is subsequently transferred to the simulation and results in a large difference between the free and total concentration scenarios. Similar effects can be observed for the Olatunde study (Olatunde and Price Evans, 1982), where the concentrations are comparable to those in the work of El-Eraky. There is a difference of up to $30 \mathrm{~ms}$ in the $\Delta \mathrm{QTc}$ between free and total scenarios, although it this connected with an underprediction of the plasma concentration in the Olatunde study, which aligns and mitigates the PD effect and makes it less spectacular.

The obvious elements that can significantly influence the final results are the in vitro current inhibition parameters. In this study a mixture of measured and predicted $\mathrm{IC}_{50}$ values was utilized. The predicted $\mathrm{IC}_{50}$ values, by default, are biased by the QSAR model error, but even if we consider the superiority of the measured overpredicted values, it still can be a source of uncertainty. This is mainly because of the lack of standard settings for the in vitro current inhibition studies, which generate multiple results depending on the cell line, temperature, and other settings applied (Polak et al., 2012e). Methodology applied in this work, where the in vitro stud- 
ies best matching the human physiology were chosen, falsely minimizes the potential negative influence on the calculated endpoint but still may result in subsequent misprediction. It would be highly recommended to apply standard methodology for the in vitro measurements that could help minimize the inter-lab differences in the obtained results and allow for reliable in vitro-in vivo scaling. In the current study, HEK cells and currents measured in the physiological temperature were chosen as the standard in vitro system, and the results were directly transferred to mimic the in vivo situation. It would be desirable to develop a standard in vitro system, possibly based on the results from human cardiomyocytes, and then to apply scaling factors allowing for direct comparison with the results obtained with the use of other cellular systems. This problem indicates a need for widening of the measured membrane currents disrupted by the drugs. The additional currents that should be assessed include potassium $\left(\mathrm{I}_{\mathrm{Ks}}\right)$, sodium $\left(\mathrm{I}_{\mathrm{Na}}\right)$, and calcium $\left(\mathrm{I}_{\mathrm{Ca}}\right)$ as potentially the most important from a drug safety assessment point of view.

One of the focal points of the study was to assess whether the utilized set of IVIVE systems is able to recover the interindividual variability. The results are generally satisfactory; in this situation, however, systems tend to slightly underpredict the plasma concentration and to a higher degree the QTc values. A viable explanation for this underprediction may lie both in the data defining the physiological parameters used during the virtual population random pick and in the characteristics of the clinical studies. One of the most important factors would be plasma ion concentrations, which undergo diurnal fluctuation that were not accounted for during the simulation. Secondly, the left ventricular heart wall thickness measurement used in this study was taken from the model proposed by Sjögren more than 40 years ago (Sjögren, 1971), and since then the quality of the analytical methods used for wall thickness measurement have changed significantly. It also could be expected that the intracellular ion concentrations differ between individuals, although such a factor was not considered due to the lack of data, and a constant value was used. Additionally, there is a 30-year period between the first (1977) and the last (2007) relevant study, in which time the healthy volunteers inclusion/exclusion criteria could have changed, which will subsequently influence the "real" versus "virtual" individual characteristics.

It is known that quinidine can influence the beta-adrenergic system and subsequently modify the heart rate (Darbar et al., 2001). There are other physiological parameters affecting the ECG characteristics that are likely to be modified by drugs. For example, the plasma ion concentration, which, following the circadian rhythms, also can be disrupted by drugs (Sennels et al., 2012). The fact that none of these effects was taken into account during this study should be considered. This is, however, a direct consequence of the main study assumption - to utilize only the in vitro data. All additional information regarding the drug-physiology relationship, regardless of the source (i.e., first-in-human studies), could be implemented and thus improve the - already accurate - predictivity.

\section{Conclusions}

According to the ICH guidelines for drug studies, cardiac safety testing in animal models is widely utilized during the testing phase ( $\mathrm{ICH}, 2005)$. This study proposes a novel concept based on a combination of mechanistic PBPK/PD modeling and simulation, which could prove invaluable in the prediction of the cardiac effects of drugs and thus help to incorporate the 3Rs concept into practice by waiving the current animal studies. The presented results illustrate reliable QTc and $\triangle \mathrm{QTC}$ prediction by the combination of the mechanistic IVIVE of the PK and PD effects. It can be considered as a proof-of-concept that could also be applied as a reliable drug safety evaluation procedure.

\section{References}

Belz, G. G., Aust, P. E., Doering, W., et al. (1982). Pharmacodynamics of a single dose of quinidine during chronic digoxin treatment. Eur J Clin Pharmacol 22, 117-122.

Ching, M. S., Elliott, S. L., Stead, C. K., et al. (1991). Quinidine single dose pharmacokinetics and pharmacodynamics are unaltered by omeprazole. Aliment Pharmacol Ther 5, 523-531.

Darbar, D., Fromm, M. F., Dellorto, S., and Roden, D. M. (2001). Sympathetic activation enhances QT prolongation by quinidine. J Cardiovasc Electrophysiol 12,9-14.

El-Eraky, H. and Thomas, S. H. (2003). Effects of sex on the pharmacokinetic and pharmacodynamic properties of quinidine. Br J Clin Pharmacol 56, 198-204.

Fieldman, A., Beebe, R. D., and Sing Sum Chow, M. (1977). The effect of quinidine sulfate on QRS duration and QT and systolic time intervals in man. J Clin Pharmacol 17, 134139.

Glinka, A. and Polak, S. (2012). Wild type and K897T polymorphisms of the hERG gene: modeling the AP in Caucasians. Bioinformation 8, 1062-1065.

ICH (2005). International Conference on Harmonization of Technical Requirements for Registration of Pharmaceuticals for Human Use. The Non-Clinical Evaluation of The Potential for Delayed Ventricular Repolarization (QT Interval Prolongation) by Human Pharmaceuticals S7B. http://www.fda. gov/RegulatoryInformation/Guidances/ucm129121.htm

Kang, J., Chen, X. L., Wang, L., and Rampe, D. (2001). Interactions of the antimalarial drug mefloquine with the human cardiac potassium channels KvLQT1/minK and HERG. $J$ Pharmacol Exp Ther 299, 290-296.

Kaukonen, K. M., Olkkola, K. T., and Neuvonen, P. J. (1997). Itraconazole increases plasma concentrations of quinidine. Clin Pharmacol Ther 62, 510-517.

Kirsch, G. E., Trepakova, E. S., Brimecombe, J. C., et al. (2004). Variability in the measurement of hERG potassium channel inhibition: effects of temperature and stimulus pattern. $J$ Pharmacol Toxicol Meth 50, 93-101.

Laganiere, S., Davies, R. F., Carignan, G., et al. (1996). Pharmacokinetic and pharmacodynamic interactions between diltiazem and quinidine. Clin Pharmacol Ther 60, 255-264. 
Lalonde, R. L., Kowalski, K. G., Hutmacher, M. M., et al. (2007). Model-based drug development state of the art. Clin Pharmacol Ther 82, 21-32.

Marshall, S., Macintyre, F., James, I., et al. (2006). Role of mechanistically-based pharmacokinetic/pharmacodynamic models in drug development: a case study of a therapeutic protein. Clin Pharmacokinet 45, 177-197.

Michel, D., Wegener, J. W., and Nawrath, H. (2002). Effects of quinine and quinidine on the transient outward and on the Ltype $\mathrm{Ca}\left(2^{+}\right)$current in rat ventricular cardiomyocytes. Pharmacology 65, 187-192.

Min, D. I., Ku, Y. M., Geraets, D. R., and Lee, H. (1996). Effect of grapefruit juice on the pharmacokinetics and pharmacodynamics of quinidine in healthy volunteers. J Clin Pharmacol $36,469-476$

Mirams, G. R., Cui, Y., Sher, A., et al. (2011). Simulation of multiple ion channel block provides improved early prediction of compounds' clinical torsadogenic risk. Cardiovasc Res 91, 53-61.

Niederer, S. A., Fink, M., Noble, D., and Smith, N. P. (2009). A meta-analysis of cardiac electrophysiology computational models. Exp Physiol 94, 486-495.

O'Hara, T. and Rudy, Y. (2011). Arrhythmia formation in subclinical ("silent") long QT syndrome requires multiple insults: quantitative mechanistic study using the KCNQ1 mutation Q357R as example. Heart Rhythm 9, 275-282.

Olatunde, A. and Price Evans, D. A. (1982). Blood quinidine levels and cardiac effects in white British and Nigerian subjects. Br J Clin Pharmacol 14, 513-518.

Polak, S. and Fijorek, K. (2012). Inter-individual variability in the pre-clinical drug cardiotoxic safety assessment - analysis of the age - cardiomyocytes electric capacitance dependence. J Cardiovasc Transl Res 5, 321-332.

Polak, S., Wisniowska, B., Ahamadi, M., and Mendyk, A. (2011). Prediction of the hERG potassium channel inhibition potential with use of Artificial Neural Networks. App Soft Comp 11, 2611-2617.

Polak, S., Wisniowska, B., Fijorek, K., et al. (2012a). ToxComp - in vitro-in vivo extrapolation system for the drugs proarrhythmic potency assessment. Comput Cardiol 39, 789-793.

Polak, S., Fijorek, K., Glinka, A., et al. (2012b). Virtual population generator for human cardiomyocytes parameters. In silico drug cardiotoxicity assessment. Toxicol Mech Methods 22, 31-40.

Polak, S., Wisniowska, B., Glinka A., et al. (2012c). Slow delayed rectifying potassium current (IKs) - analysis of the in vitro inhibition data and predictive model development. $J$ Appl Toxicol Feb 14. doi: 10.1002/jat.2719.

Polak, S., Wisniowska, B., Glinka, A. and Polak, M. (2012d). tox-database.net - data describing the chemicals triggered in vitro cardiac ionic channels inhibition. BMC Pharmacol Toxicol 2012, 13.
Rescigno, A. (1992). Bioequivalence. Pharm Res 9, 925-928.

Rostami-Hodjegan, A. and Tucker, G. T. (2007). Simulation and prediction of in vivo drug metabolism in human populations from in vitro data. Nat Rev Drug Discov 6, 140-148.

Sennels, H. P., Jørgensen, H. L., Goetze, J. P., and Fahrenkrug, J. (2012). Rhythmic 24-hour variations of frequently used clinical biochemical parameters in healthy young males - The Bispebjerg study of diurnal variations. Scand J Clin Lab Invest 72, 287-295.

Shin, J. G., Kang, W. K., Shon, J. H., et al. (2007). Possible interethnic differences in quinidine-induced QT prolongation between healthy Caucasian and Korean subjects. Br J Clin Pharmacol 63, 206-215.

Sjögren, A. L. (1971). Left ventricular wall thickness determined by ultrasound in 100 subjects without heart disease. Chest 60, 341-346.

ten Tusscher, K. H., Noble, D., Noble, P. J., and Panfilov, A. V. (2004). A model for human ventricular tissue. Am J Physiol Heart Circ Physiol 286, H1573-H1589.

Wisniowska, B., Mendyk, A., Fijorek, K., et al. (2012). Predictive model for the L-type channel inhibition - multichannel block in QT prolongation risk assessment. J Appl Toxicol 32, 858-866.

Woolf, S. H. (2008). The meaning of translational research and why it matters. JAMA 299, 211-213.

Zerhouni, E.A. (2005). Translational and clinical science - time for a new vision. $N$ Engl J Med 353, 1621-1623.

\section{Acknowledgements}

I would like to acknowledge Dr Ruth Clayton from Simcyp Ltd. for professional help during manuscript preparation and Prof. Amin Rostami-Hodjegan from School of Pharmacy, University of Manchester, UK for his advice and comments on the manuscript. This project was financed by Polish National Center for Research and Development LIDER project number LIDER/02/187/L-1/09.

\section{Correspondence to}

Sebastian Polak, PhD

Unit of Pharmacoepidemiology and Pharmacoeconomics,

Faculty of Pharmacy

Jagiellonian University Medical College

Medyczna 9 Street

30-688 Krakow

Poland

Phone: +48 126205517

Fax: +48 126205519

e-mail: spolak@cm-uj.krakow.pl 